\title{
Topology Optimization of Acetabular Cup by Finite Element Simulation
}

\author{
M. A. AbDullah
}

University of Debrecen, Faculty of Engineering, Department of Basic Technical Studies, m.ahmed@eng.unideb.hu University of Debrecen

Abstract: Hip replacements typically consist of a four-part piece. Our research will focus primarily on the acetabular component. Several different types of materials can be used when creating a hip replacement implant ranging from plastic to titanium. Different materials are used to accommodate for allergic reactions or circumventing potential health risks. Aside from the material, the size of the components plays a factor in terms of durability; a larger diameter head might avoid dislodgement though it could increase wear and tear on the stems through constant friction. A patient's force applied to the hip replacement is usually measured through a number of physical assessments. Finite element analysis (FEA), a computer-based method of data observation, allows for us to accurately simulate hip forces and their impact on the hip replacements. Through this, it becomes easier to predict and calculate the performance of specific designs. Generative systems can also be used to support performance analysis and optimization through assessing a multitude of cases, many of which apply in real-world scenarios. By applying both systems, we designed and modeled an acetabular cup that when measured decreased the mass from 129 grams initially down to 52 grams, a 60\% decrease in total mass. Furthermore, the design we created lessened the trauma on the piece through distributing force across the entirety of the piece rather than specific segments only. This shows an increased durability and life expectancy when compared to usual acetabular cups.

\section{Introduction}

The acetabulo-femoral joint, usually known as hip joint, is the joint between the femur and acetabulum of the pelvis and it supports the weight of body as its major role in both static (i.e., standing) and dynamic (e.g. running or walking) postures. The hip joints have very fundamental roles in maintaining balance, and for sustaining the pelvic inclination angle.

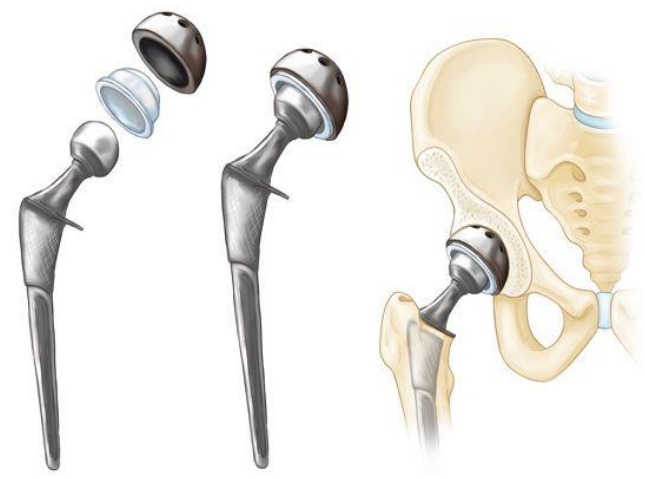

Figure 1. Overview of Hip Implant 
There are many reasons of hip pain which may include osteoarthritis, nervous, infectious, genetic and trauma-related. The hip joint' is a synovial joint formed by the articulated movement of the rounded head of the femur and the cup-like pelvic acetabulum. It provides connection between the lower limb bones and the axial skeleton of the pelvis and trunk. Articular hyaline cartilage covers both joint surfaces which is a strong and also a lubricated layer.

The cup shaped acetabulum forms at the point where three pelvic bones unite - the ilium, pubis, and ischium [1]. The Y-shaped growth plate separates them, the triradiate cartilage, gets fused definitively at ages 14-16 [2]. It is a type of ball and socket or spheroidal joint where the femoral head (roughly spherical) is placed within the acetabulum and its average radius of curvature is $2.5 \mathrm{~cm}$. [3] The acetabulum grabs almost half of the femoral ball, a grip supported by a ring shaped fibrocartilaginous lip called the acetabular labrum which extends the joint beyond the equator. [4] The joint space which is between the superior acetabulum and the femoral head is usually between 2 and $7 \mathrm{~mm} .[5]$

\section{The Components: What Makes Up a Hip Replacement Implant?}

A natural hip joint is made up of two main components: the ball and socket. During a complete hip arthroplasty, four parts are introduced to create a new hip. The four artificial components are:

a) The acetabular component

b) A plastic liner

c) A femoral head and

d) The femoral stem.

1.1. Acetabular component (socket): It is a bowl-shaped piece which represents new socket. This bowl or cup shaped piece is fit into resurfaced socket. This piece is normally made up of metal but it is seldom made up of ceramic or by combining both metal and plastic.

1.2. Acetabular liner: It is normally made up of a high-quality plastic. The acetabular component, which is basically a plastic liner that fits into the socket, allows the movement of femoral head (ball) in a very smooth, easy and more natural way in the socket.

1.3. Femoral head (ball): The ball attached firstly into the new, plastic lined socket and is then fits into the femoral stem. There are many sizes and shapes of heads. These are made of longlasting metal, ceramic, plastic, or by amalgamation of other material.

1.4. Femoral stem: The stem then connected to the ball and provides support to novel hip joint. Typically, the piece of this metal is porous which allows natural or original bone to grow and join to this piece which replaces femur. 

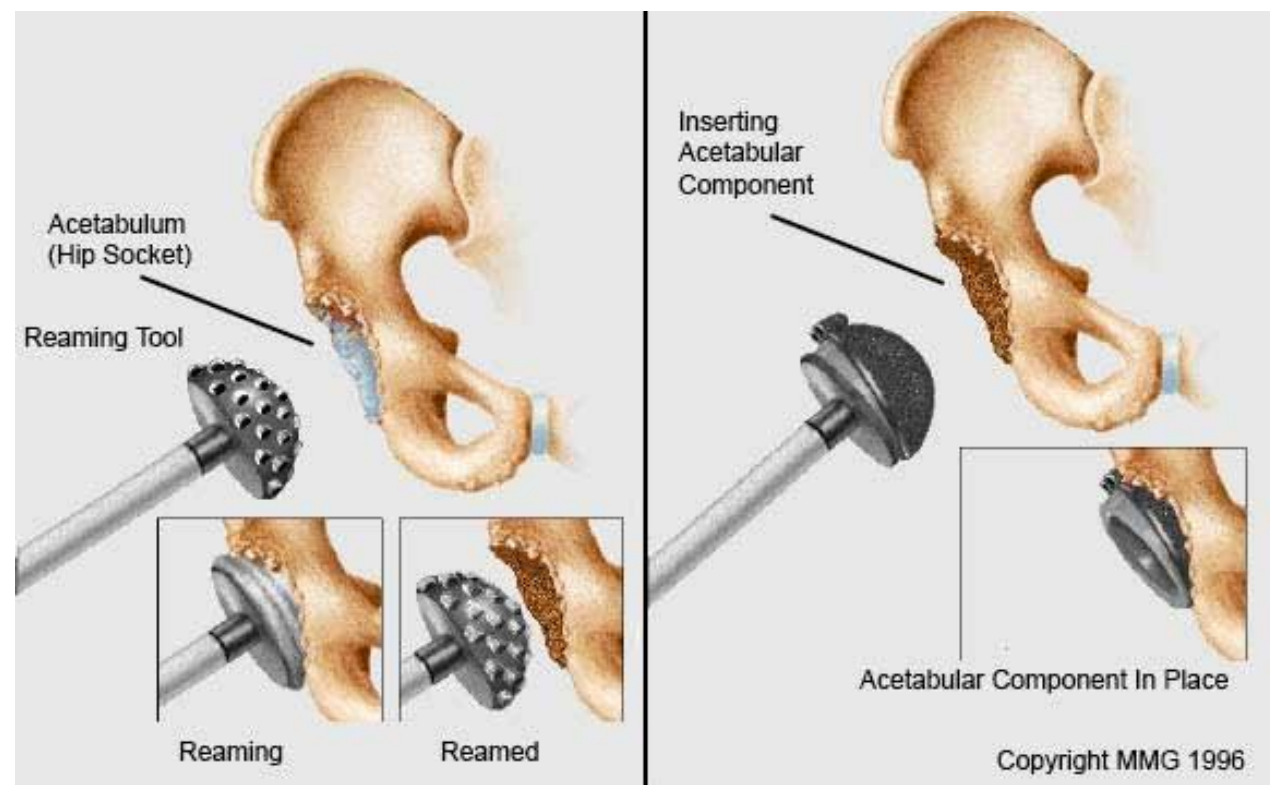

Figure 2. Installation of Hip Implants

\section{What are materials that can be used in hip replacement?}

Strong plastic, metal or ceramic are the materials that can be used in synthesis of artificial replacement parts. Titanium, titanium cobalt, stainless steel, cobalt-chromium alloys, or titanium and cobalt mixed metals are used in the composition of femoral stem component in most cases. For the composition of head, liner and acetabular parts, either metal, plastic, ceramic or combination of above can be used. Implant materials must be as strong as flexible allowing it to move on regular basis. They also must be appropriate for the body and won't cause any reactions (biocompatible).

\subsection{Standard Implant Component Materials}

\subsubsection{Metal on Metal (MOM)}

When both the socket and the ball components are made up of metal it is termed as metal on metal component (MOM). The amalgamation of metals like cobalt chromium alloys, cobalt mixed metals or titanium can be used in the composition of these metals.

\subsubsection{Polyethylene and Metal on Polyethylene (MOP)}

Polyethylene can be defined as a plastic which is a metal free and is of high quality. Usually this plastic can be utilized in the composition of socket or acetabular. Furthermore, this plastic can be utilized to cover other components that are made up of metals. MOP can be considered when socket is made up of plastic and ball is made up of metal.

\subsubsection{Ceramic on Metal (COM), Ceramic on Polyethylene (COP), Ceramic on Ceramic (COC)}

Usually, ceramic hips are not frequently used metal as it is less common that is why it is not utilized by all surgeons. Ceramic material is used for those people who have allergic issues with metals. It is commonly used in the amalgamation with special metal parts or plastic components. Even though, 
ceramic parts are long lasting but historically in comparative to other metal components, ceramic metals have been more brittle. However, as the time passing these metals are also changing. Currently, ceramic parts are disputed to outlast metal part.

Those who have allergic issues with metals used titanium alloy as it is very soft metal. Titanium alloy is very soft metal with very low amounts of nickel. There are many components of replacements that are $100 \%$ free from metals. When you are in doubt to start to have allergic issues, immediately consult your care team and ask to get tested and for proper treatment.

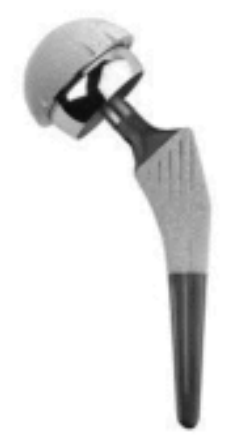

Figure 3. A metal-on-metal femoral stem and cup

\section{Historical background}

In 1925, the American surgeon Marius Smith-Petersen produced the first mold arthroplasty out of glass. It can be fit over the femoral head to gives us a new smooth surface for movement as it is consisted of hollow hemisphere. Regardless of glass being a biocompatible material it becomes shattered and could not endure the great forces that are going through the hip. Marius Smith, Petersen and Philip Wiles used current stainless material to prepare the first total hip replacement that fitted to bone with screws and bolts. [3] [4]

George McKee, an English surgeon, was the first person who used a metal-on-metal prosthesis on a regular basis. By using the modified Thompson stem (a cemented hemiarthroplasty which is used for neck of femur fracture treatment) he began with a new one-piece cobalt-chrome socket as the new acetabulum in 1953. This prosthesis had a good survival rate as a recent study shows a 28 year survival rate of 74 percent. [5] Until now, this method became unpopular by the mid 1970's because of local effects of metal particles seen during revision surgery for prosthetic failure. [6]

Sir John Charnley, the orthopaedic surgeons considered the father of the modern THA, the one who worked at the Manchester Royal Infirmary. The prostheses, we used today is identical to his low friction arthroplasty designed in the early 1960's. Low friction arthroplasty had three parts viz a polyethylene acetabular component, a metal femoral stem and acrylic bone cement - that were borrowed from dentists. [7] Because small femoral head had smaller surface area that is why Charnley advocated the utilization of a small femoral head which lessens wear, so it was named as the low friction arthroplasty. The most commonly used and systematically followed up of all the prosthesis is Metal-on-polyethylene (M-on-PE), making up the majority of THA undertaken in the UK today. [8] Popularized in 1970s, by the early success of the Charnley prosthesis, implants that are 
polyethylene based are almost completely displaced all other bearing surfaces, so much so that a huge quantity of research was intended to develop a design and to improve implantation techniques purely for the M-on-PE prosthesis. 10 At this time, most of the patient were provided by a safe , predictable and cost effective bearing and for many it represented the gold standard in THA. [9]

In 1970s after falling out of favour, Metal-on-metal (M-on-M) prostheses started to experience a restoration. Previously, concerns were elevated about the bearings potential to produce metal ions, the process of metallises, which had a speculative carcinogenic risk, as well as associated prosthetic loosening and hypersensitivity reactions. It is believed that the cause for aseptic loosening in first generation models was due to poor design and improper implantation technique rather than the Mon-M bearings themselves.

Prosthetic wear in M-on-M reported to be sixty times less than expected with conventional M-onPE prostheses. [10] Besides, the metal femoral heads can have a larger diameter, increasing joint stability as they are less fragile than other materials and hence the incidence of dislocation in these arthroplasties is lesser. [11] M-on-M implants also reduce osteolysis and peri-prosthetic inflammatory tissue as compared to its polyethylene counterpart. [12]

\section{Femoral head size}

The size of the femoral head normally ranges from $40 \mathrm{~mm}$ to $54 \mathrm{~mm}$ naturally and in females' smaller sizes usually are found. Artificial femoral heads were designed to copy these anatomical dimensions in the early development of hip arthroplasty. Though, it very soon became clear that large diameter metallic heads are the cause of high amounts of polyethylene wear debris. Providing a solution to this problem, [13] introduced the successful use of $22 \mathrm{~mm}$ diameter metal heads, and this trend of using femoral heads which are smaller than the ones found naturally, continues today. However, there were some differences and developments in response to further concerns raised by the use of small femoral heads. In the 1970s faintly larger femoral heads, about $28 \mathrm{~mm}$, were introduced to neutralize the issue of dislocation attributable to the smaller femoral heads [14]. The evolution of the femoral head design sizes are shown in Fig. 4.

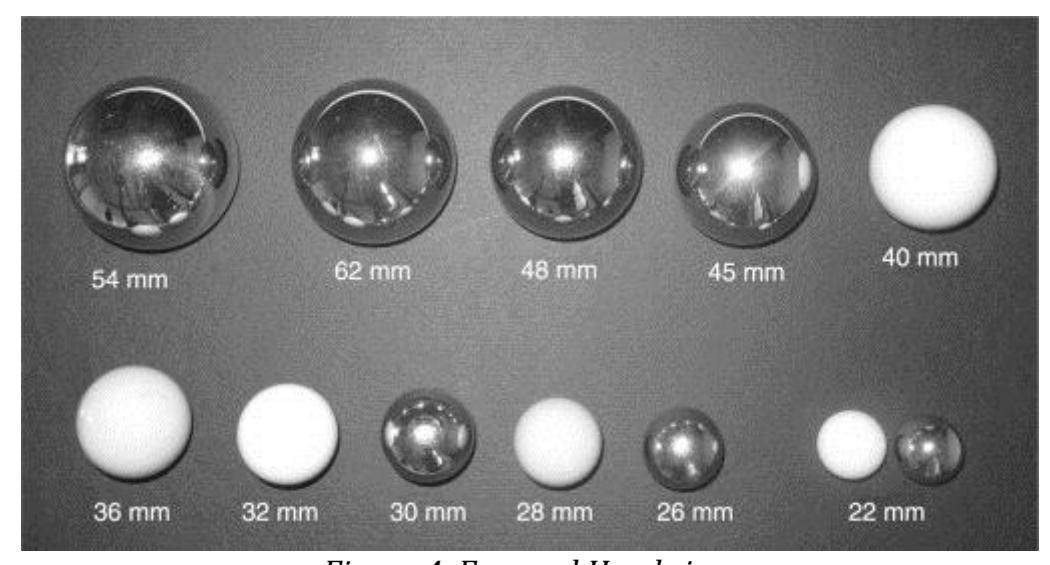

Figure 4. Femoral Head sizes 
Many studies showed that the probabilities of dislocation incidents can be decreased by using large ball therefore; dislodgment of the femoral heads is a key concern in modern THA. In addition, minimizing the limitations given by hip joint arthroplasty has become a main problem to think about in response to the expectations and activities of young patients. The progress of new materials and modern production methods has once again made the use of larger femoral heads (32, 36 and $40 \mathrm{~mm})$, using ceramics and metals, practicable.

\subsection{Femoral head replacement material}

Femoral heads are generally prepared with ceramic material or metals, Co-Cr-Mo-cast alloys, stainless steel and alumina (pure or BIOLOX delta), or zirconia are the most commonly used materials for this purpose. The material and design should provide guarantee for mechanical resistance to the tensile stresses that increase along the taper junction. Ceramic femoral heads mark a higher surface smoothness than metal femoral heads, meaning that lower friction rates are reachable. But, ceramic femoral heads can only be produced with a maximum diameter of about 38 to $40 \mathrm{~mm}$ because of the limitations in the manufacturing technology whilst the low toughness and high hardness create the risk of brittle fractures for the ceramic material. [15]

In the last decade, perfections in THA devices have allowed surgeons to shift towards the utilization of larger femoral head diameters, which better match the internal structure of the healthy hip joint. [16] By more strictly matching the anatomy, such devices offer improved stability against dislocation and a wider range of motion that results in an improved quality and number of the physical activities, can be achieved by the patients.

\subsection{Acetabular liner replacement material}

However, the multi-component nature of THA devices must be taken into account, alongside such developments. MOP or COP prostheses having large diameter femoral heads do not fit so well because the greater sliding distance and the higher sliding velocity of that of the bearing surfaces enhance the UHMWPE wear. Additionally, wider outer and inner diameters need of the acetabular liner (for UHMWPE and ceramic) will end in a lower shell thickness, as the physical space for insertion is determined by the internal structure of the pelvis. This will ultimately lead to lower fixation quality and therefore lower mechanical stability. Due to this reason, soft materials and brittle materials cannot be taken into use as liners when using large diameter heads. Thus when shell and the liner are combined to form a single piece to provide necessary mechanical strength then these types of devices need metallic acetabular cup liners or mono-block acetabular cups. The only successful material combination available currently for large diameter head hip joint prostheses is MOM coupling. [17] Ceramic-on-UHMWPE (Ultra High Molecular Weight Poly-Ethylene) is a good mixture of two reliable materials. Ceramic heads are harder as compared to metal and these are the largely scratch resistant implant material. The ultra-smooth and solid surface can significantly minimize the wear rate for the polyethylene bearing. For this type of implant, the possible wear rate is less than Metal-on-Polyethylene (MOP). 


\subsection{Force required}

Force required is derived from activity measurements among patients having hip implants [18], these includes without using the handrail moving down or up a staircase, at self-selected speed walking on level ground, standing in a one-legged posture and standing up or sitting down. Slow jog at $7 \mathrm{~km} / \mathrm{hour}$ was as well observed, as many enjoy this athletic activity and patients progressively perform it even after they had been through hip replacement [19]. Moreover, cycling is regularly exercised by patients with implants, at a reasonable power expenditure of $90 \mathrm{~W}$, as an alternative to walk for a long distance or as an activity for fitness [20]. Both activities may be executed with an enhanced number of loading cycles and that is why they may have a potential control on implant fatigue. As in ISO standards it was described that most endurance tests are currently performed, of the implant stem, by applying unidirectional sinusoidal forces. If this practice is supposed to be continued, then the amplitude and direction of the test force should be adjusted to realistic HIGH 100 forces. One probability could be to combine the highest activity amplitude of $\mathrm{F}_{\text {res }}$ (3875 $\mathrm{N}$ in stair descent) by the average force directions $\alpha_{\text {front }}$ and $\alpha_{\text {sagit }}$ from the most insisting activity (ascending/descending stairs) and the most common activity (walking), which would be $13.5^{\circ}$ and $17.5^{\circ}$ respectively. If only walking should be simulated, a maximum force of $\mathrm{F}_{\text {res }}=2880 \mathrm{~N}$ would have to act at $\alpha_{\text {front }}=17^{\circ}$ and $\alpha_{\text {sagit }}=11^{\circ}$.

Titanium implant that was clinically proven (Berlin, Merete Medical, CTW \& Germany) with a ceramic head of $32 \mathrm{~mm}$ that moved with a press-fit cup with XPE inlay was customized to measure the moments and forces exerting at the head. The electronics influenced inductively by a coil around the hip joint, in the void implant neck was equipped with a 9-channel amplifier with telemetric data shift and six strain gauges. The electronics were encapsulated carefully by welding. This method gives us a real time monitoring of the 3D forces and seconds with an accuracy of 1-2 percent.

\section{Introduction of Finite Element Analysis (FEA)}

The Finite Element Analysis (FEA) is an arithmetic method that solves problems of mathematical physics and engineering. It is helpful in solving problems with difficult geometries, material properties and loadings where systematic solutions cannot be obtained.

Finite element analysis (FEA) is totally computer-based method for calculating how a real-world object will react to heat, forces, vibration, etc. It is called analysis in terms of whether it will wear out, break or will work the way it was devised whereas in the product design cycle it issued to predict what will happen when the product is used. The restricted element procedure is violation of real object into a large number (about 1,000s to 100,000s) of elements such as tiny cubes. Each little element's behaviour which is regular in shape is predicted via set mathematical equations. Then computer adds up all of the individual behaviours to analyse the behaviour of the real object.

\subsection{The Rationale of FEA Analytical Solution}

FEA is used for stress analysis for beams, trusses, and other simple structures are carried out that are based on dramatic idealization and simplification, mass concerted at the centre of gravity and beam 
that are simplified as a line segment (same cross-section). Its design is founded on the results of calculation of the idealized structure and a large protective factor (1.5-3) given by experience. FEA Design geometry is more complex and demands a lot higher accuracy. There is a need to be aware of the physical behaviours of a difficult object (heat transfer capability, strength, fluid flow, etc.) Along with that we need to predict or calculate the behaviour and performance of the design. [21]It is used to spot the faults of the design accurately, predict the safety margin and to make out the optimal design with assurance.

\subsection{Application}

It can be applied to many areas including mechanical, aerospace, civil, automotive engineering, structural, stress analysis, static/dynamic linear/nonlinear, fluid flow, heat transfer, electromagnetic fields, soil mechanics and acoustics biomechanics.

\section{EFA}

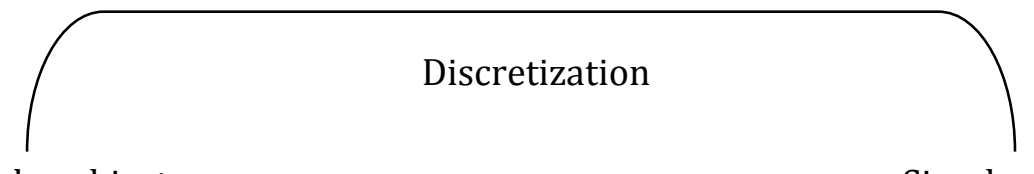

Complex object $\quad$ Simple analysis

(Material discontinuity, Comfaceplex \& Arbitrary geometry)

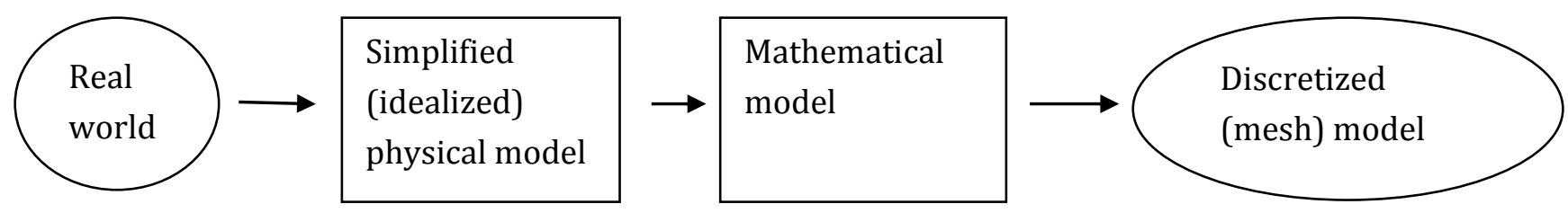

\subsubsection{Discretization}

Model body by dividing it into a corresponding system of numerous units or smaller bodies (finite elements) that are unified at points common to two or more elements (nodes or nodal points) and/or surfaces and/or boundary lines.

\subsubsection{Feature}

Its features find a set of algebraic equations that gives us solution firstly for unknown nodal quantity which is displacement and secondary quantities that are stresses and strains and then are expressed in terms of nodal values of primary quantity.

\subsubsection{Principles of FEA}

The Finite Element Method (FEM) or Finite Element Analysis (FEA), is a computerized based technique that is used to acquire estimated solutions regarding boundary value issues in engineering. Boundary value problems are also termed as field problems. The field most frequently characterizes a physical structure and is the area of interest. The field variables are dependent variables of interest 
administered by differential equation. The boundary circumstances are the particular values of the field variables or related variables, such as derivatives, on the boundaries of the field.

\subsubsection{Shape functions}

Field variable's values are computed at the nodes generally used to estimate the values at non-nodal points i.e., in the element interior, by exclamation of nodal values. For the three-node triangle example, the field variable is expressed by the approximate relation $\varphi(x, y)=N 1(x, y) \varphi 1+N 2(x, y) \varphi 2+N 3(x$, y) $\varphi 3$ where $\varphi 1, \varphi 2$, and $\varphi 3$ are the values of the field variable at the nodes And N1, N2, and N3 are the outburst functions which are also named as shape functions or blending functions. In the finite or restricted element approach, the nodal values of the field variable are treated as unknown constants that are to be determined. The interpolation or outburst functions are most common polynomial shapes of the independent variables that are obtained to satisfy certain conditions which are required at the nodes. The interpolation or outburst functions are identified functions of the independent variables, predetermined; and these functions explain the discrepancy of the field variable within the finite element.

\section{Generative design}

Soddu, Celestino defines generative design as a "morphogenetic process that uses algorithms structures as nonlinear system for endless unrepeatable and unique results performed by an ideacode, as in nature" [22]. Definitely, the notions of digital morphogenesis and generative designs are strongly connected. The term 'morphogenesis' derived from Greek words, morphe meaning 'form' and term genesis meaning 'birth', so it could literally mean 'birth of form'. As with the term generative design, there is no exceptional definition for morphogenesis, and it seems that the terminology keeps on changing in relation to emerging techniques and technologies.

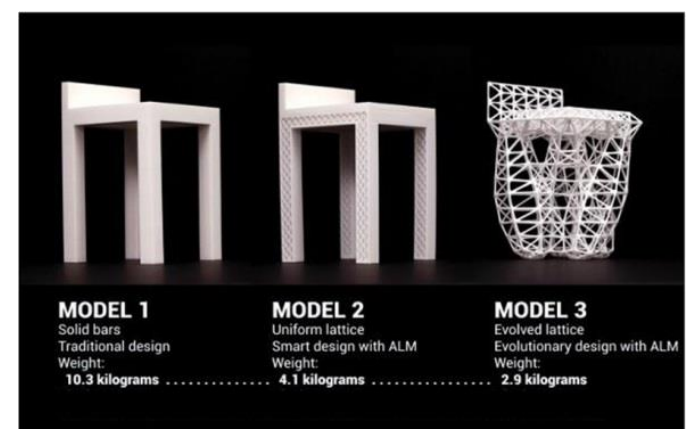

Figure 5. Generative design model (copied from google)

In order to truly support and accomplish the needs of the design process, design tools must embrace change. Generative Design is defined by the foundation of shapes that are determined by algorithms. When these algorithms are interpreted in a programming language and defined parametrically, they automatically embrace change. Generative design uses algorithms, goals and parameters (forces, fixed points etc) and helps us to decrease the mass, it increases the efficiency, product size or geometric dimensions, materials, manufacturing methods, cost per unit. Generative design is stronger. Generative Systems (GS) may be used to support performance analyses and optimization, automatic 
extraction of documentation, mass customization strategies and direct fabrication through Computer Numerical Controlled (CNC) machines.

Generative design uses algorithms, goals and parameters (forces, fixed points etc) and helps us to:

- Decrease the mass

- Increase the efficiency

- product size or geometric dimensions

- materials

- manufacturing methods

- cost per unit

- Stronger

\subsection{Simulation and Optimization}

- Solid Edge Simulation is built-in finite element analysis (FEA). Computer Aided Design (CAD) simulations helps us in many ways, some of them are used to reduce the need for physical prototypes to save time and money.

- Verify if assemblies are overbuilt and based on the amount of stress that is exerted on them. Determine the load for which a structure loses its stability.

- Analyse heat conditions on mechanical or electro-mechanical assemblies.

\subsection{D model}

We have created three different models for acetabular cup as shown below:

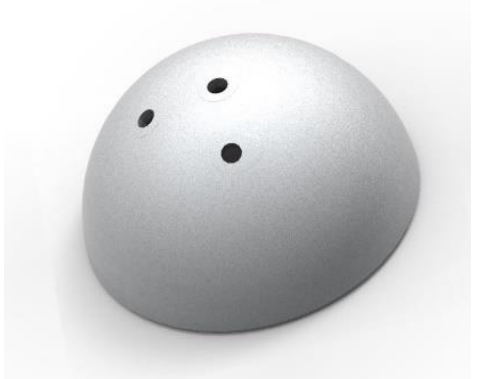

Figure 6. Three-hole cup

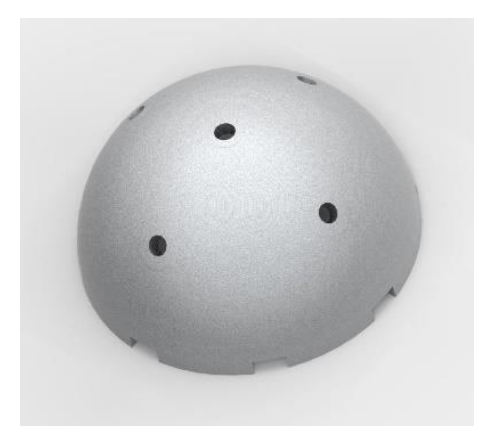

Figure 7. Five Hole Cup

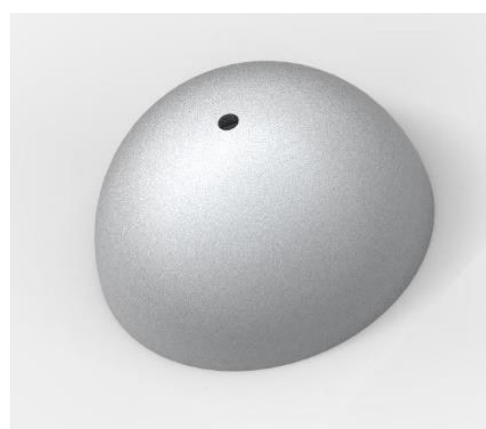

Figure 8. One Hole Cup

Due to certain complications we decided to go on with three holes acetabular cup. Five hole and one hole were complicated for the simulation as there were no supportive fixed nodes for the cantered hole. We will show the results of five-hole cup in results section. 


\subsection{Properties}

We used about 700 newtons of torque and $0.7 \mathrm{KPa}$ of pressure on the inner surface of the cup and fixed the holes for screws (the data is taken from a journal).

- Titanium, unalloyed

- Stainless steel

- Aluminium, 1060

- Galvanized steel

\section{Results}

\subsection{Result 1}

By using generative design, we were able to find some acceptable results which does not have any complication for health issues either. Two results are shown below, one for five hole and three-hole acetabular cup.
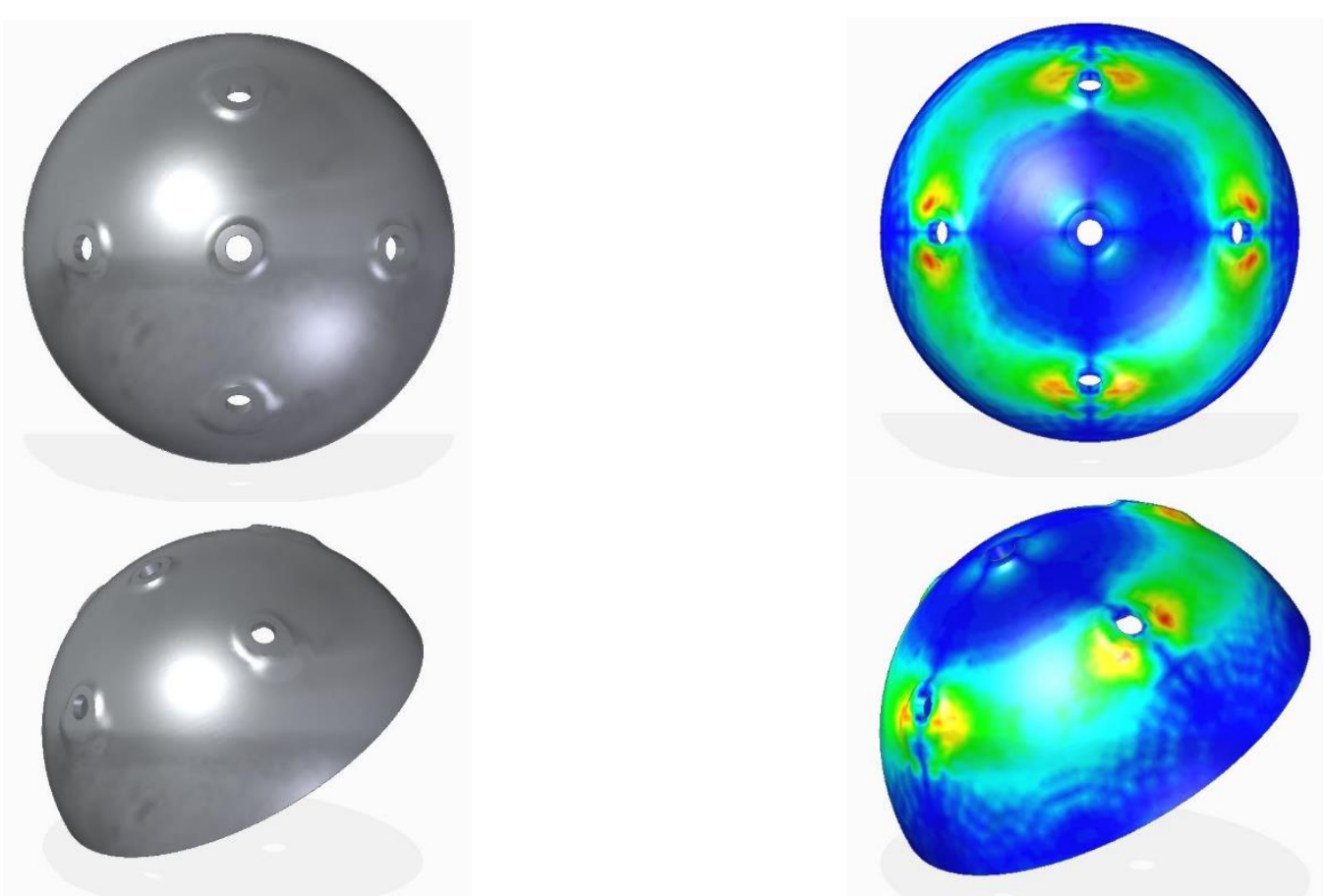

Figure 9. Result 1 for five hole cup

Original mass was 108 grams which was reduced to 50 grams. Which is $54 \%$ of the total mass. We can see a lot of stress around the holes which is deformable / wearable in a period of time. 


\subsection{Results 2}
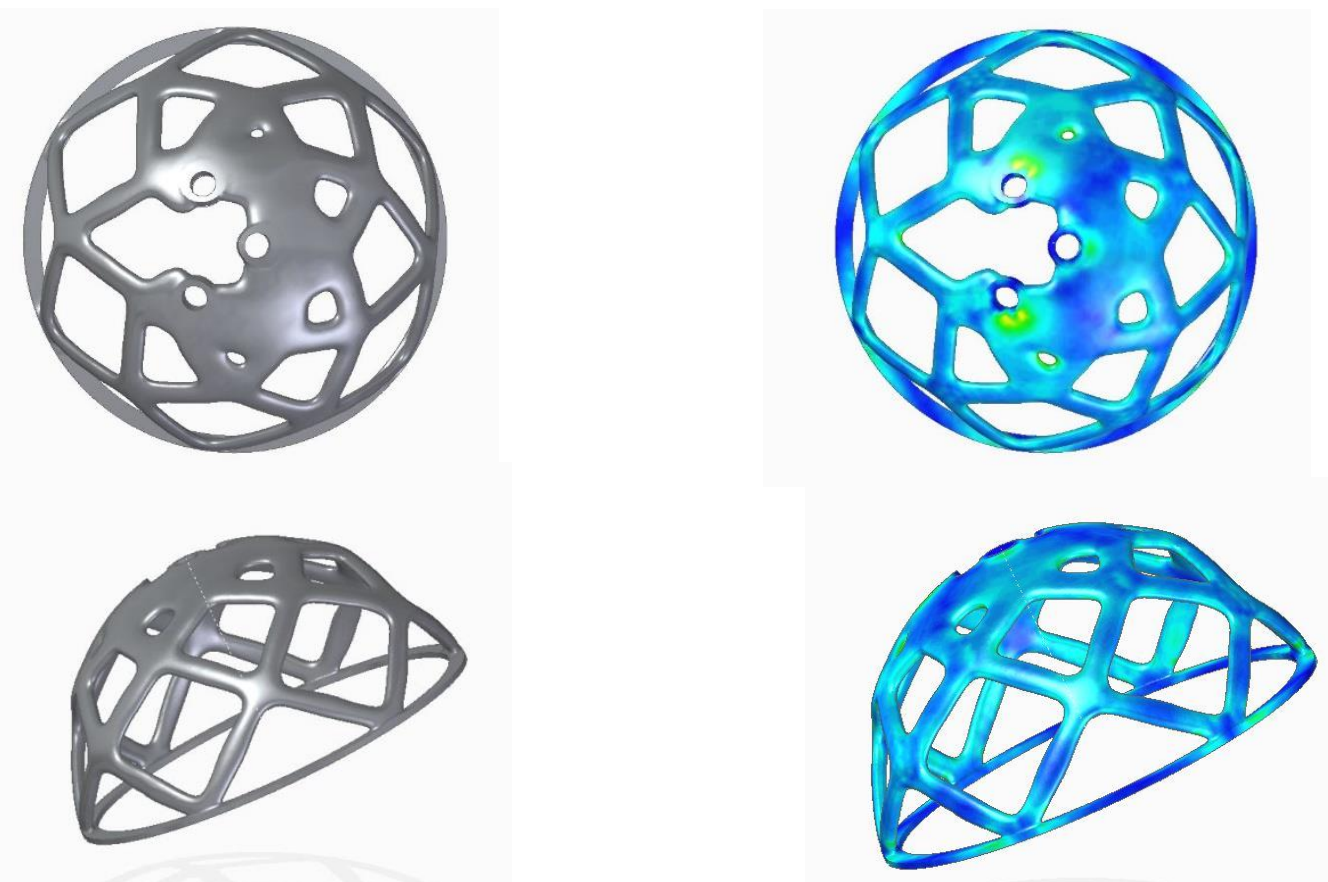

Figure 10. Result 2 Three-hole

With the help of generative design, we decreased the mass originally from 129 grams to 52 grams, which is $60 \%$ of the total mass. There are not too much stresses around the holes or anywhere around the cup. Which shows us its durability and life expectancy can be a lot more than usual.

\section{References}

[1] L. B. Hôpital, "The normal hip joint space: variations in width, shape, and architecture on 223 pelvic radiographs," Annals of the Rheumatic Diseases., pp. 174-175, 2006.

[2] H. Sandhu, Middleton and RG, "Thieme Atlas of Anatomy," Google Scholar, p. 74, 2006.

[3] M. Smith-Petersen, "The classic: evolution of mould arthroplasty of the hip joint," J Bone Joint Surg., p. 308, 1948.

[4] P. Wiles, "The surgery of the osteo-arthritis hip.," Br J Surg., p. 45, 1957.

[5] S. Brown, W. Davies, H. DeHer and A. Swanson, "Long-term survival of Mckee-Farrar total hip prosthesis. Clin Orthop Relat Res.," Google Scholar, p. 157, 2002.

[6] H. McKellop, S. Park and R. Chiesa, "In vivo wear of three types of metal on metal hip prostheses during two decades of use.," Clin Orthop Relat Res., p. 329, 1961.

[7] J. Charnley, "Arthroplasty of the hip: a new operation.," Lancet, p. 32, 1961.

[8] N. Nakamura, "he NHS Information Centre. Hospital Episode Statistics for England.," Inpatient statistics,, [Online]. Available: www.hesonline.nhs.uk.. 
International Journal of Engineering and Management Sciences (IJEMS) Vol. 5. (2020). No. 2

DOI: 10.21791/IJEMS.2020.2.4.

[9] H. Sandhu and R. Middleton, "Controversial topics in orthopaedics: ceramic-on-ceramic.," Ann R Coll Surg Engl, p. 87, 2005.

[10] J. Cuckler, "The rationale for metal-on-metal total hip arthroplasty.," Google Scholar, p. 174, 2005.

[11] M. Archibeck, J. Jacobs, K. Roebuck and T. Glant, "The basic science of periprosthetic osteolysis.," Google Scholar, pp. 85-88, 2001.

[12] P. Boutin, Total arthroplasty of the hip by fritted aluminum prosthesis. Experimental study and 1st clinical applications., 1972.

[13] J. Cuckler, "The rationale for metal-on-metal total hip arthroplasty.," Clin Orthop Relat Res., pp. 132-139, 2005.

[14] M. Archibeck, J. Jacobs, K. Roebuck and T. Glant, "The basic science of periprosthetic osteolysis," Instr Course Lect., pp. 50-95, 2001.

[15] P. Boutin, "Total arthroplasty of the hip by fritted aluminum prosthesis. Experimental study and 1st clinical applications.," Rev Chir Orthop Reparatrice Appar Mot., pp. 46-58, 2012.

[16] M. Tamás, "Numerical analysis of unit cell models for orthopedic applications," IOP CONFERENCE SERIES: MATERIALS SCIENCE AND ENGINEERING, p. 393, 2018.

[17] Holzwarth and Cotogno, "Total hip arthroplasty," Google Scholar, p. 92, 2012.

[18] M. Morlock, E. Schneider, A. Bluhm, M. Vollmer, G. Bergmann and V. Muller, "Duration and frequency of every day activities in total hip patients.," Journal of biomechanics., p. 34, 2001.

[19] H. Abe, T. Sakai, T. Nishii, M. Takao, N. Nakamura and N. Sugano, "Jogging after total hip arthroplasty.," The American journal of sports medicine., p. 42, 2014.

[20] M. D. C, "Which type of exercise therapy is effective after hip arthroplasty? A systematic review of randomized controlled trials.," Eur Physics Rahabil Med, p. 49, 2016.

[21] M. Tamás, A. V. Tamás, M. Sándor and K. Imre, "Compressive Response Determination of Closed-Cell Aluminium Foam and Linear-Elastic Finite Element Simulation of $\mu C T$-Based Directly Reconstructed Geometrical Models," STROJNISKI VESTNIK-JOURNAL OF MECHANICAL ENGINEERING, pp. 105113, 2018.

[22] C. Soddu, Morphogenetic algorithms, 1994. 